\title{
Effectiveness of percutaneous laser revascularization therapy for refractory angina
}

\author{
This article was published in the following Dove Press journal: \\ Vascular Health and Risk Management \\ 24 August 2010 \\ Number of times this article has been viewed
}

\author{
Michael McGillion' \\ Allison Cook ${ }^{2}$ \\ J Charles Victor ${ }^{3}$ \\ Sandra Carroll ${ }^{2}$ \\ Julie Weston' \\ Kevin Teoh ${ }^{4}$ \\ Heather M Arthur ${ }^{2}$ \\ 'University of Toronto, ${ }^{3}$ Dalla Lana \\ School of Public Health, Toronto, \\ ON, Canada; ${ }^{2}$ McMaster University, \\ Faculty of Health Sciences, Hamilton, \\ ON, Canada; ${ }^{4}$ Hamilton Health \\ Sciences, Hamilton, ON, Canada
}

Correspondence: Michael McGillion University of Toronto, Suite I30, I 55 College Street, Toronto, ON, Canada, M5T IP8

$\mathrm{Tel}+$ I 4169463989

Fax + I 4169788222

Email michael.mcgillion@utoronto.ca
Abstract: Refractory angina is a debilitating disease characterized by persistent cardiac pain resistant to all conventional treatments for coronary artery disease. Percutaneous myocardial laser revascularization (PMLR) has been proposed to improve symptoms in these patients. We used meta-analysis to assess the effectiveness of PMLR versus optimal medical therapy for improving angina symptoms, health-related quality of life (HRQL), and exercise performance; the impact on all-cause mortality was also examined. Seven trials, involving a total of 1,213 participants were included. Our primary analyses showed that at 12-month follow-up, those who had received PMLR had $\geq 2$ Canadian Cardiovascular Society class angina symptom reductions, OR 2.13 (95\% CI, 1.22 to 3.73), as well as improvements in aspects of HRQL including angina frequency, SMD $=0.29(95 \% \mathrm{CI}, 0.05$ to 0.52$)$, disease perception, $\mathrm{SMD}=0.37$ (95\% CI, 0.14 to 0.61$)$, and physical limitations, $\mathrm{SMD}=0.29(95 \%$ CI, 0.05 to 0.53$)$. PMLR had no significant impact on all-cause mortality. Our secondary analyses, in which we considered data from one trial that featured a higher-dose laser group, yielded no significant overall impact of PMLR across outcomes. While PMLR may be effective for improving angina symptoms and related burden, further work is needed to clarify appropriate dose and impact on disease-specific mortality and adverse cardiac events.

Keywords: refractory angina, percutaneous laser revascularization, angina symptoms, health-related quality of life, meta-analysis

\section{Introduction}

Refractory angina (RFA) is a debilitating disease characterized by persistent cardiac pain. ${ }^{1-3}$ This pain/discomfort, by definition, is resistant to all conventional treatments for coronary artery disease (CAD) including nitrates, calcium-channel and $\beta$ adrenoceptor blockade, vasculoprotective agents, percutaneous coronary interventions, and coronary artery bypass grafting. ${ }^{1-3}$ Patients living with RFA have a low annual mortality rate of $3 \%$ but suffer severely impaired health-related quality of life (HRQL). ${ }^{4}$ They typically experience recurrent and sustained pain, poor general health status, psychological distress, impaired role functioning, activity restriction, and inability to self-manage..$^{5-8}$ The global prevalence of RFA is increasing, likely due to improvements in revascularization techniques and patients' increased survival after primary and subsequent cardiac events. ${ }^{1,2,9,10}$

Percutaneous myocardial laser revascularization (PMLR) therapy emerged as a treatment option for RFA in the 1990s, as an alternative to transmyocardial laser revascularization (TMLR). A major impetus for adopting PMLR, across several countries, was the elimination of the incumbent risks of sternotomy and/or left anterior thoracotomy required for the TMLR procedure. ${ }^{11-13}$ PMLR entails the application of 
holmium: yttrium aluminum garnet (holmium: YAG) laser energy to the endocardial surface of the left ventricle via a flexible catheter; laser firing is synchronized during systole to create a series of nontransmural channels in targeted regions with reversible ischemia. ${ }^{11,12}$ As with TMLR, ${ }^{14}$ the mechanisms of action during PMLR are unclear. Proposed laser mechanisms with insufficient and/or contradictory evidence include direct perfusion, ${ }^{15-18}$ microvascular angiogenesis, ${ }^{19-23}$ and cardiac afferent denervation. ${ }^{24-27}$ There is also insufficient evidence to support placebo effect ${ }^{13,28}$ and change in attitudes and expectations ${ }^{24}$ towards angina as significant factors in symptom relief.

While the mechanisms of therapeutic benefit have remained in question, symptom relief, improvements in exercise duration, HRQL, and safety have been reported in several randomized controlled trials (RCTs). ${ }^{12,13,29,30-32}$ For example, Salem et $\mathrm{al}^{31}$ found sustained angina relief, without revascularization, up to 3 years post-intervention in the absence of procedural mortality, myocardial infarction (MI), or cerebral embolism; cardiac event-free survival (cardiac death, MI, stroke, transient ischemic attack, myocardial perforation, rehospitalisation) was $88 \%$ at one year and $66 \%$ at late follow-up (mean $=3$ years). ${ }^{31}$ Despite some positive findings, PMLR has since fallen out of favor in a number of centers due to ongoing controversy over mechanisms and effectiveness. Effective treatment options for RFA symptoms are, however, limited, and reviews of PMLR trial data to date have been narrative-based. A 2009 Cochrane Review clearly demonstrated that the observed clinical benefits of TMLR do not outweigh the potential perioperative risks. ${ }^{14}$ Given that PMLR is a less invasive approach, we felt that the available data on clinically relevant patient outcomes should be subjected to a level of meta-analytic rigor comparable to that of TMLR in order to offer more definitive conclusions about its effectiveness for symptom relief.

\section{Objectives}

This review aims to determine the effectiveness of PMLR plus maximal medical therapy (MMT) versus MMT alone for improving pain symptoms, HRQL, and exercise performance in patients with RFA. All-cause mortality was examined as a secondary outcome.

\section{Criteria for selection of studies included in this review}

\section{Study designs}

All published and unpublished RCTs of PMLR with parallel designs; follow-up period varied. Nonrandomized studies and single-group design studies were excluded.

\section{Participants}

Adult RFA patients 18 years or older with CAD determined angiographically or via nuclear imaging, class III or IV angina according to the Canadian Cardiovascular Society (CCS) angina classification scale, and maximally-tolerated doses of antianginal therapy, most typically including beta-blockers, calcium antagonists, and nitrates. Those patients included were also no longer eligible for conventional revascularization procedures including coronary artery bypass grafting $(\mathrm{CABG})$ and percutaneous coronary intervention (PCI) due to a) unsuitable coronary anatomy such as diffuse sclerosis and lesions not amenable to intervention (eg, unprotected left main, distal, or highly calcified), or b) extracardiac diseases resulting in unacceptable perioperative risk including (but not limited to) carotid stenosis, renal insufficiency, and chronic obstructive pulmonary disease (COPD).

\section{Types of interventions and controls}

PMLR performed via percutaneous arterial access using a holmium: YAG laser system was compared with maximallytolerated antianginal therapy. Studies included patients who may have undergone conventional revascularization procedures (CABG or PCI) prior to the study period. Studies that included additional therapies as adjuvants to PMLR, such as intramyocardial autologous bone marrow cell implantation and vascular endothelial growth factor implantation, were excluded.

\section{Outcomes}

1. Angina symptoms

2. Self-reported HRQL via generic or disease-specific instruments

3. Exercise duration

4. All-cause mortality

\section{Search methods for identification of studies}

We searched the Cochrane Central Register of Controlled Trials, MEDLINE, PubMed, CINHAL, EMBASE, Proquest Dissertation Abstracts, Psychinfo, and HealthStar, from Jan 1999-June 2009, using combinations of key medical subject heading $(\mathrm{MeSH})$ terms including refractory angina, stable angina, angina pectoris, laser therapy, laser revascularization, percutaneous laser revascularization, Holmium:YAG laser, randomized controlled trials, and clinical trials. We also conducted hand searches of relevant journals, proceedings of major conferences, and secondary references; experts in the field were consulted for additional sources. Our search strategy was critiqued and replicated by an external information specialist to ensure comprehensiveness. 


\section{Methods}

\section{Final selection of trials}

Five reviewers reached consensus on all trials to be included in this meta-analysis by reviewing the titles, abstracts, and reports of all trials according to the inclusion criteria specified a priori; individual trial results were not considered during this process.

\section{Data extraction and appraisal of methodological quality}

Four reviewers participated in independent quality assessment and extraction of process and outcome data from each trial according to a data extraction form developed for this review. The methodological quality of included trials was appraised via standard Cochrane criteria for risk of bias assessment ${ }^{33}$ including generation of randomization sequence; allocation concealment; blinding of participants, personnel, and outcome assessors (detection bias); use of sham procedure (placebo effect); standardized intervention delivery and presence of cointervention (performance bias); reliability and validity of measurement instruments (insensitive measurement bias); response rate (RR) and attrition (attrition bias); and selective reporting (reporting bias). Propensity for selection bias was also assessed. Reported outcome data were taken directly from included published reports, and consensus among the four reviewers was reached across trials on quality assessment and data extracted.

\section{Data synthesis and analysis}

Continuous outcomes were summarized using standardized mean differences (SMD). These were determined using differences in change over baseline at the end of treatment, across treatment groups, divided by the pooled standard deviation. If change over baseline was unavailable, differences in mean values at the end of treatment were used. For studies reporting only medians and interquartile ranges (IQR) for continuous outcomes, we planned to estimate means and standard deviations (SD) using the method outlined by Hozo et $\mathrm{al}^{34}$ Tomlinson and Beyene ${ }^{35}$ have suggested that omission of studies reporting only medians and IQRs may lead to a loss of important information. A SMD of 0.20 standard deviation units was considered a small difference between the experimental and control groups, a SMD of 0.50 a moderate difference, and 0.80 a large difference. ${ }^{36,37}$ Binary outcomes were expressed as odds ratios. We used standard inverse-variance random-effects meta-analysis to combine the trials. ${ }^{38}$ Heterogeneity between trials was evaluated using Chi-squared tests for the Tau-squared statistic and quantified using the $\mathrm{I}^{2}$ statistic, ${ }^{39}$ which describes the percentage of variation across trials that is attributable to heterogeneity rather than to chance. $I^{2}$ values of $25 \%, 50 \%$, and $75 \%$ may be considered as indicators of low, moderate, and high heterogeneity, although this has been shown to depend on the size and number of trials included. ${ }^{40}$ In instances where significant heterogeneity might be found, we planned to conduct sensitivity analyses by removing studies, such as those with estimated mean values or those of lower methodological quality, in order to determine factors related to the heterogeneity and the effect on the pooled outcome.

\section{Description of studies}

Seven trials, ${ }^{12,13,28-30,32,41}$ conducted in 3 countries and published in peer-reviewed journals between 2001 and 2006 , met the criteria for inclusion in this review. The total number of patients was 1,213; 651 were randomly allocated to the PMLR group. All studies employed a holmium:YAG laser in their respective intervention groups. Catheter systems used to deliver laser energy varied, including the ECLIPSE ${ }^{29,41}$ the Coaxial Cardiogenesis System, ${ }^{13,30}$ the Axcis PMLR, ${ }^{12,32}$ and the Biosense direct myocardial revascularization system. ${ }^{28}$ The number of laser channels formed ranged from 8-35 per patient, depending on location and extensiveness of ischemic territory, proximity to cardiac structures, and maneuverability of the catheter. Descriptions of the operative protocols including femoral arterial access, anticoagulation regimen, and sedation were available and similar across 6 of the 7 trials. Techniques used to map ischemic territories were described in 6 trials and included fluoroscopy and biplane ventriculography and coronary angiography in orthogonal views, ${ }^{12,13,28-30,32,41}$ left ventricular electromechanical mapping, and single-photon-emission computed tomography (SPECT).$^{28}$ Across trials, no changes were made to participants' maximally-tolerated antianginal therapy in PMLR and control groups.

The characteristics of each trial included are presented in Table 1. Sample sizes ranged from 68-330, and all patients had CCS Class III-IV angina. One trial included patients with a single chronic total occlusion (CTO) and failed PCI. ${ }^{41}$ All trials compared sociodemographic and baseline measures between groups; no significant differences were found (level of detail varied) with the exception of one trial wherein a higher proportion of patients with hyperlipidemia, family history of CAD, previous cardiac interventions, and a higher median Seattle Angina Questionnaire (SAQ) score was found in the control group. ${ }^{12}$ All trials excluded patients 
Table I Characteristics of included trials

Study
Design
Relevant outcomes, definitions
Sample size, participant characteristics, setting, country
Measurement occasions, outcome measures, reliability and validity

Intervention

Notes

Study

Design

Relevant outcomes, definitions

Sample size, participant characteristics, setting, country

Measurement occasions, outcome measures, reliability and validity

Intervention

Notes

Study

Design

Relevant outcomes, definitions

Sample size, participant characteristics, setting, country

Measurement occasions, outcome measures, reliability and validity

Intervention

Notes

Study

Design

Relevant outcomes, definitions

Sample size, participant characteristics, setting, country

Measurement occasions, outcome measures, reliability and validity

Intervention

Notes

Study

Design

Relevant outcomes, definitions

Sample size, participant characteristics, setting, country

Measurement occasions, outcome measures, reliability and validity
Gray et al $2003^{30}$

RCT

Angina severity, Exercise duration, QoL,

$\mathrm{N}=73$ (M-70/F-3), RFA patients, CCS class III-IV, MMT, unsuitable for revascularization single UK center, $2 \mathrm{I}$ of this sample were also analyzed in Oesterle et al, 2000

Change in exercise duration, CCS, McGill pain score and SAQ at $3,6,12$ mths from baseline, (reliable and valid tools) change in LVEF from baseline to $3 \mathrm{mths}$ as assessed on echocardiograph PMLR plus MMT compared to MMT

Coaxial Cardiogenesis ${ }^{\mathrm{TM}}$ PMLR laser system

Leon et al $2005^{28}$ DIRECT Trial

RCT

Exercise tolerance, angina frequency, QoL, morbidity, mortality, myocardial perfusion

$\mathrm{N}=298$, (m-229/f-69), RFA patients, CCS class III-IV, MMT, unsuitable for revascularization, 14 US Centers

Change in exercise duration from baseline to $6 \mathrm{mths}$ using modified Bruce protocol, MACE at I, 6, 12 mths, procedural adverse events at $30 \mathrm{~d}$, change in CCS class from baseline to 6 and 12 mths, SF-12, SAQ from baseline to 6 and 12 mths, change in radionuclide perfusion scores from baseline to $6 \mathrm{mths}$

Three arm trial comparing low-dose laser vs high-dose laser vs medical management

Holmium:YAG laser with Biosense DMR TM catheter

McNab et al $2006^{32}$ SPiRiT Trial

RCT

Exercise tolerance, angina severity, QoL, adverse events, myocardial perfusion

$\mathrm{N}=69$ (M-60/F-8), RFA patients, CCS class III-IV, MMT, unsuitable for revascularization, UK tertiary referral center

Change in exercise treadmill time using modified Bruce protocol, CCS class, SF-36, SAQ, and Euroqol from baseline at 3 and 12 mths procedural and disease-related adverse events documented reliability and validity of tools

PMLR compared to intermittent spinal cord stimulation

Axcis PTMR TM Holmium:YAG laser

\section{Oesterle et al $2000^{12}$ PACIFIC Trial}

RCT

Exercise duration, angina severity, survival, death, MI, hospitalization $N=22$ I (M-I90/F-3I), RFA patients, CCS class III-IV, MMT, unsuitable for revascularization, 12 US and I UK centers Change in exercise duration, CCS and SAQ at 3, 6, 12 mths from baseline (reliable and valid tools)

PMLR plus MMT compared to MMT

Axcis PTMR ${ }^{\text {TM }}$ Holmium:YAG laser system, 24 patients received revascularization procedures during the one year follow-up, analysis was done by ITT and also excluding this group

\section{Salem et al $2004^{13}$ Belief Trial}

RCT

Exercise tolerance, angina severity, QoL

$\mathrm{N}=82$ (M-75/F-7), RFA patients, CCS class III-IV, MMT, unsuitable for revascularization, 2 Norwegian centers

Change in CCS, SAQ, medication usage, from baseline to 6 and 12 mths, chronotropic assessment exercise protocol used to assess change in exercise time, oxygen uptake, and respiratory exchange ratio from baseline to 12 mths 
Table I (Continued)

\begin{tabular}{|c|c|}
\hline Study & Salem et al $2004^{13}$ Belief Trial \\
\hline Intervention & PMLR plus MMT vs MMT \\
\hline Notes & $\begin{array}{l}\text { Coaxial Cardiogenesis }{ }^{\mathrm{TM}} \text { Holmium:YAG system, LVEF reported } \\
\text { unchanged but no description of instrument used, blinding of all } \\
\text { personnel, participants and outcome assessors for } 12 \text { mths }\end{array}$ \\
\hline Study & Stone et al $2002^{41}$ \\
\hline Design & $\mathrm{RCT}$ \\
\hline Relevant outcomes, definitions & Adverse events, 6 month cumulative MACE \\
\hline Sample size, participant characteristics, setting, country & $\begin{array}{l}\mathrm{N}=14 \mid \text { (M-I I2/F-29), RFA patients, CCS class III-IV, MMT, } \\
\text { unsuitable for revascularization, I7 US centers }\end{array}$ \\
\hline Measurement occasions, outcome measures, reliability and validity & $\begin{array}{l}\text { Change in exercise duration with Bruce protocol and CCS from } \\
\text { baseline to } 6 \text { and } 12 \text { mths, difference in MACE between groups }\end{array}$ \\
\hline Intervention & PMLR vs MMT \\
\hline Notes & $\begin{array}{l}\text { Enrolled after failed attempt at } \mathrm{PCI} \text {, no description of randomization } \\
\text { process, only procedure room staff aware of group allocation, } \\
\text { Eclipse }^{\mathrm{TM}} \text { Holmium:YAG system }\end{array}$ \\
\hline Study & Whitlow et al $2003^{29}$ \\
\hline Design & $\mathrm{RCT}$ \\
\hline Relevant outcomes, definitions & Exercise duration, angina severity, QoL \\
\hline Sample size, participant characteristics, setting, country & $\begin{array}{l}\mathrm{N}=330 \text { (M-245/F-85) RFA patients, CCS class III-IV,MMT, unsuitable } \\
\text { for revascularization }\end{array}$ \\
\hline Measurement occasions, outcome measures, reliability and validity & $\begin{array}{l}\text { Change in exercise tolerance (Naughton protocol) and from baseline } \\
\text { to } 12 \mathrm{mths} \text {, change in CCS, DASI from baseline to } 6 \text { and } 12 \text { mths }\end{array}$ \\
\hline Intervention & PMLR plus MMT vs MMT \\
\hline Notes & Eclipse $^{\mathrm{TM}}$ Holmium:YAG laser system, no patient blinding \\
\hline
\end{tabular}

Abbreviations: CCS, Canadian Cardiovascular Society; d, days; DASI, Duke Activity Status Index; LVEF, left ventricular ejection fraction; MACE, major adverse cardiovascular event; MI, myocardial infarction; MMT, maximum medical therapy; mths, months; PCl, percutaneous coronary intervention; PMLR, percutaneous myocardial laser revascularization; QoL, quality of life; RFA, refractory angina; SF-12, Medical Outcome Study: General Health Survey; SF-36, Medical Outcomes Study: 36 Item Short Form; SAQ, Seattle Angina Questionnaire; YAG, yttrium-aluminum-garnet.

with a recent MI, aortic stenosis, mechanical aortic valve, peripheral vascular disease precluding the insertion of a 9 French arterial access catheter, diminished left ventricular (LV) function (ejection fraction $<25 \%-30 \%$ ), and myocardial wall thickness less than $8-9 \mathrm{~mm}$ in the laser-targeted region(s). Additional exclusion criteria in some trials included clinically significant ventricular arrhythmias, ${ }^{12,13,30,41}$ left ventricular thrombus, ${ }^{12,13,28-30,41}$ pacemakers and defibrillators, ${ }^{32}$ and chronic atrial fibrillation. ${ }^{28}$

Relevant outcome measures in the trials reviewed are presented in Table 2. All trials examined the impact of PMLR on angina pain symptoms and exercise tolerance. The CCS classification system was used to measure angina pain across trials; one trial ${ }^{30}$ also used the McGill Pain Questionnaire. Measures of exercise performance included the modified Bruce protocol, ${ }^{12,28,30,32,41}$ the Naughton protocol, ${ }^{29}$ and treadmill or bicycle ergometry. ${ }^{13}$ Six trials reported HRQL outcomes using various measures including the $\mathrm{SAQ},{ }^{12,13,28,30,32}$ the Short Form (SF)-12, ${ }^{28}$ the Short Form (SF)-36, ${ }^{32}$ the EuroQoL, ${ }^{32}$ and the Duke Activity Status Index (DASI). ${ }^{29}$ Adverse events and mortality during follow-up were reported across trials; means to capture these data included cumulative major adverse cardiac events (MACE) including death, MI and revascularization, ${ }^{12,28,41}$ freedom from MACE, ${ }^{12,13,28,29}$ event-free survival, ${ }^{13}$ periprocedural ${ }^{28-30,12}$ and vascular ${ }^{12,32}$ complications, hospital admissions, ${ }^{12,13,30}$ increase in antianginal medications, ${ }^{12,13,30}$ and medical complications, including cerebral vascular accident (CVA), transient ischemic attack (TIA), heart failure, and arrhythmias..$^{12,30}$ Length of follow-up period for data collection ranged from 3-12 months, varying by outcome and rate of attrition.

\section{Risk of bias in included studies}

Details of our risk of bias assessment are provided in Figures $1 \mathrm{a}$ and $1 \mathrm{~b}$. One trial by Gray et $\mathrm{al}{ }^{30}$ was excluded from metaanalysis due to a number of methodological shortcomings. First, clinical results were presented for 73 patients using trial procedures from the PACIFIC trial. ${ }^{12}$ While these procedures included a process of randomization, no a priori sample size calculation or stopping rules were stated. Second, analyses were conducted on an ongoing basis and recruitment was stopped when sufficient evidence was available from both this and the PACIFIC trial to make decisions about the continued use of PMLR. Finally, 21 participants in Gray et al's 
Table 2 Outcomes relevant to this review reported by authors

\begin{tabular}{|c|c|c|c|c|}
\hline Trial & Angina pain symptoms & Exercise duration & HRQL & Adverse events \\
\hline \multirow[t]{9}{*}{ Gray et al $2003^{30}$} & CCS class, & Modified Bruce protocol & SAQ & Hospital admissions \\
\hline & McGill pain questionnaire & & & Cerebral vascular accident \\
\hline & & & & Transient ischemic attack \\
\hline & & & & Other periprocedural and vascular \\
\hline & & & & complications \\
\hline & & & & Hospital admissions \\
\hline & & & & Arrythmias \\
\hline & & & & Heart failure \\
\hline & & & & Increase in antianginal medication \\
\hline \multirow[t]{4}{*}{ Leon et al $2005^{28}$} & CCS class & Modified Bruce protocol & SAQ & MACE \\
\hline & & & SF-I2 & LV perforation \\
\hline & & & & Stroke \\
\hline & & & & Other periprocedural complications \\
\hline \multirow[t]{3}{*}{ McNab et al $2006^{32}$} & CCS class & Modified Bruce protocol & EuroQOL & Vascular complications \\
\hline & & & SAQ & All-cause mortality \\
\hline & & & SF-36 & \\
\hline \multirow[t]{9}{*}{ Oesterle et al $2000^{12}$} & CCS class & Modified Bruce protocol & SAQ & MACE \\
\hline & & & & Cerebral vascular accident \\
\hline & & & & Transient ischemic attack \\
\hline & & & & Other periprocedural and vascular \\
\hline & & & & complications \\
\hline & & & & Hospital admissions \\
\hline & & & & Arrythmias \\
\hline & & & & Heart failure \\
\hline & & & & Increase in antianginal medication \\
\hline \multirow[t]{5}{*}{ Salem et al $2004^{13}$} & CCS class & Bicycle ergometry & SAQ & LV Function \\
\hline & & & & Event-free survival \\
\hline & & & & Hospital admissions \\
\hline & & & & MACE \\
\hline & & & & Increase in antianginal medication \\
\hline \multirow[t]{2}{*}{ Stone et al $2002^{41}$} & CCS class & Modified Bruce protocol & & MACE \\
\hline & & & & Periprocedural complications \\
\hline \multirow[t]{2}{*}{ Whitlow et al $2003^{29}$} & CCS class & Naughton protocol & Duke activity & Periprocedural complications \\
\hline & & & status index & MACE \\
\hline
\end{tabular}

trial were also participants in the PACIFIC trial; and since outcome data were not separated by trial, this precluded their use for meta-analysis.

The methodological quality of the remaining six trials ranged from moderate to high. We considered 4 to be of high quality ${ }^{12,13,28,32}$ and 2 of moderate quality. ${ }^{29,41} \mathrm{~A}$ number of reports did not adequately describe the randomization process (sequence generation and allocation concealment) but none used methods that were of obvious poor quality. Blinding of outcome assessors was clearly reported in 5 of the 6 trials $(83 \%)$. Blinding of the patients and of the personnel caring for them occurred in $4(66 \%)$ and $3(50 \%)$ of the trials respectively. A sham procedure was used in 3 trials (50\%). Loss-to-follow-up rates ranged from 0\%-12\% across the 4 trials of high methodological quality. ${ }^{12,13,28,32}$ Of the remaining two trials, the loss-to-follow-up rate was $45 \%$ in one ${ }^{41}$ and presumably $0 \%$ in the other ${ }^{29}$ as no losses-to-follow-up were reported. Five trials ${ }^{12,13,28,29,32}$ reported on all prespecified outcomes at all time points. In the remaining trial by Stone et $\mathrm{al}^{41}$ all prespecified outcomes were reported at 3 and 6 months, but prespecified 12-month data were not included. This trial was also excluded from our meta-analysis because of inadequate reporting and the high loss-to-follow-up rate $(45 \%)$.

\section{Effects of interventions}

The impact of PMLR was evaluated at 12 months post-intervention across outcomes measured. The DIRECT trial $^{28}$ included three arms: a sham procedure, low-dose (10-15 pulses) and high-dose (20-25 pulses) laser treatment groups, respectively. Since this trial found no significant differences in the effects of high- and low-dose laser treatment we therefore decided a priori to conduct our primary meta-analysis using data from the low-dose laser group in order to be conservative. As a form of sensitivity analysis, we conducted secondary meta-analyses for each outcome 

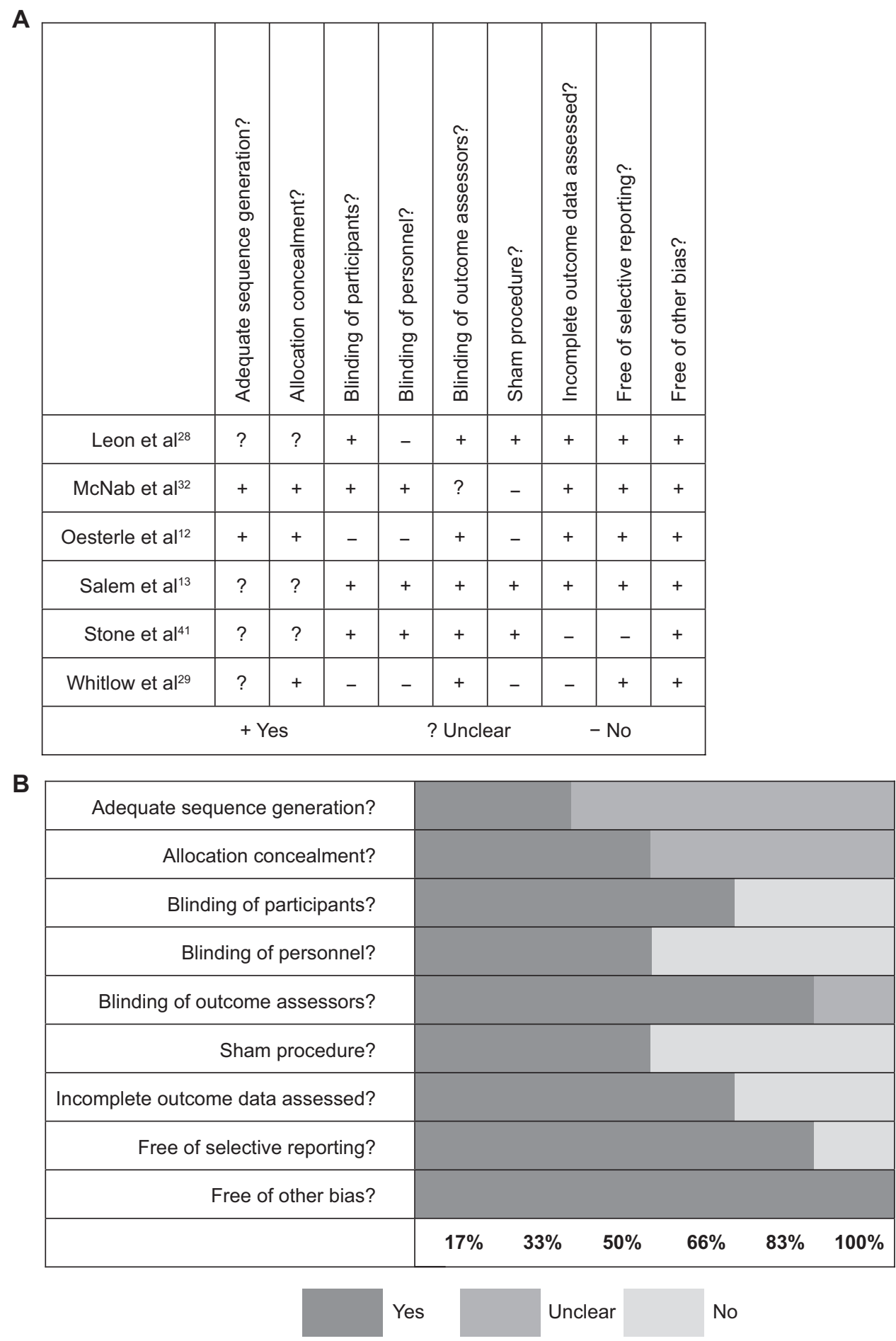

Figure I Risk of bias assessment of included studies expressed as A) yes/no/unclear, and B) percentage.

using data from the high-dose group in order to identify any possible changes in the magnitude of our pooled estimates of effect.

With respect to our primary meta-analysis, the PACIFIC trial ${ }^{12}$ was a significant source of heterogeneity (ie, $\mathrm{I}^{2}>50 \%$ ) across all outcomes apart from mortality. This was likely due to the fact that summary data in the PACIFIC trial were expressed as medians and interquartile ranges (IQR), requiring estimation of means and standard deviations (SD). (Our original inclusion of PACIFIC trial data did not result in any significant shift in the direction of magnitude of pooled estimates of effect.) Details of outcomes pertaining to CCS class, HRQL, and exercise duration are therefore based on sensitivity analyses which ultimately excluded PACIFIC trial data. 


\section{Primary analyses CCS class}

Data pertaining to changes in CCS class were available in three trials. ${ }^{13,28,29}$ We examined the impact of PMLR on change in CCS class of two categories or more, as this approach was standard across included trials. Two of the three trials showed a significant reduction in at least 2 angina classes; ORs ranged from 1.32 to 2.74 , with a pooled OR of 2.13 (95\% CI 1.22 to 3.73) (Figure 2), demonstrating an overall significant impact of PMLR on CCS class $(P=0.008)$.

\section{Health-related quality of life}

Extractable data were available from two trials, ${ }^{13,28}$ that examined the impact of PMLR on self-reported HRQL with the disease-specific SAQ. The SAQ quantifies five clinically relevant domains of disease-specific HRQL including: angina frequency, angina stability, disease perception, physical limitation, and treatment satisfaction; no overall functional status summary score is derived. ${ }^{42}$ As seen in Figures 3, 4, and 5 results of our meta-analyses showed significant improvements in angina frequency [SMD $=0.29,95 \% \mathrm{CI}, 0.05$ to $0.52, P=$ 0.02], disease perception [SMD $=0.37,95 \% \mathrm{CI}, 0.14$ to $0.61 . P$ $=0.002]$, and physical limitation $[\mathrm{SMD}=0.29,95 \% \mathrm{CI}=0.05$ to $0.53, P=0.02]$. No significant improvements in angina stability or treatment satisfaction were found (Figures 6 and 7).

\section{Exercise performance}

Exercise performance, measured as exercise duration in seconds, was examined using data from three trials. ${ }^{13,28,29}$ Two trials showed no significant increase in exercise duration, with SMDs of -0.04 and 0.15 respectively. An overall pooled SMD across trials of $0.23,95 \%(\mathrm{CI}=-0.06$ to 0.53$)$, found no significant difference between treatment and control groups regarding exercise duration $(P=0.12)$ (Figure 8$)$.

\section{Mortality}

Mortality and adverse event-related data were available from 4 trials (including the PACIFIC trial). ${ }^{12,13,28,29}$ Event-free survival data were reported via Kaplan-Meier curves or proportions; reporting of mortality data in combination with adverse outcomes varied across trials precluding a detailed approach to a) patient survival versus the occurrence of adverse events, and b) disease-specific mortality. We therefore examined all-cause mortality using absolute number of deaths reported. No trials showed significant differences between treatment and control groups, with ORs ranging from 0.33 to 3.05 . PMLR was not found to have a significant overall impact on all-cause mortality: pooled OR, 1.34 (95\% CI 0.44 to 4.03 ,
$P=0.61)$; our test for heterogeneity was not significant $\left(\mathrm{Chi}^{2}\right.$ $: 2.66, P=0.45, \mathrm{I}^{2}=0 \%$ ) (Figure 9).

\section{Secondary analyses}

As a form of sensitivity analysis, meta-analyses were repeated using data from the DIRECT Trial ${ }^{28}$ high-dose laser group as opposed to the low-dose laser group. The results of these secondary analyses are presented in Table 3. When the high-dose laser group data were used, no significant differences between treatment and control groups, across outcomes, were found.

\section{Discussion}

In this review we have appraised the summarized 12-month outcomes of 5 trials, conducted in 3 countries, of PMLR versus maximal medical therapy for RFA management; data from 2 of the original 7 trials reviewed were excluded due to poor methodological quality. The search strategy to identify these trials was comprehensive for PMLR-specific interventions with a restriction of publication in the English language. All trials reviewed included patients with refractory angina characterized by CCS class III or IV symptoms no longer amenable to conventional revascularization procedures and treated with maximally-tolerated antianginal therapy. The methodological quality of the 5 trials ranged from moderate to high, with 5 trials blinding outcome assessors, 4 trials blinding participants, and 3 trials blinding PMLR operators and other clinicians involved.

With respect to our primary analyses, PMLR was found to significantly reduce angina by at least two CCS classes and significantly improve three dimensions of self-reported HRQL measured via the SAQ including: angina stability, angina-induced physical limitations, and disease perception. Pooled SMDs for these SAQ subscales ranged from 0.29 to 0.37 suggesting a small difference ${ }^{36,37}$ in the positive impact of PMLR plus maximal medical therapy (ie, treatment) versus maximal medical therapy alone (ie, control). Taken together, despite the small SMDs, these results, with the significant improvements found in CCS class, are encouraging, considering the high levels of perceived psychological burden and related disability known to be associated with unrelieved CCS Class III-IV angina symptoms..$^{3-8}$ Our results are consistent with findings of a recent Cochrane Review of TMLR ${ }^{14}$ where those who underwent TMLR experienced significant reductions in CCS angina class and significant improvement in perceived physical limitation as compared to controls receiving optimal antianginal medical therapy. 
Experimental Control Odds ratio Odds ratio

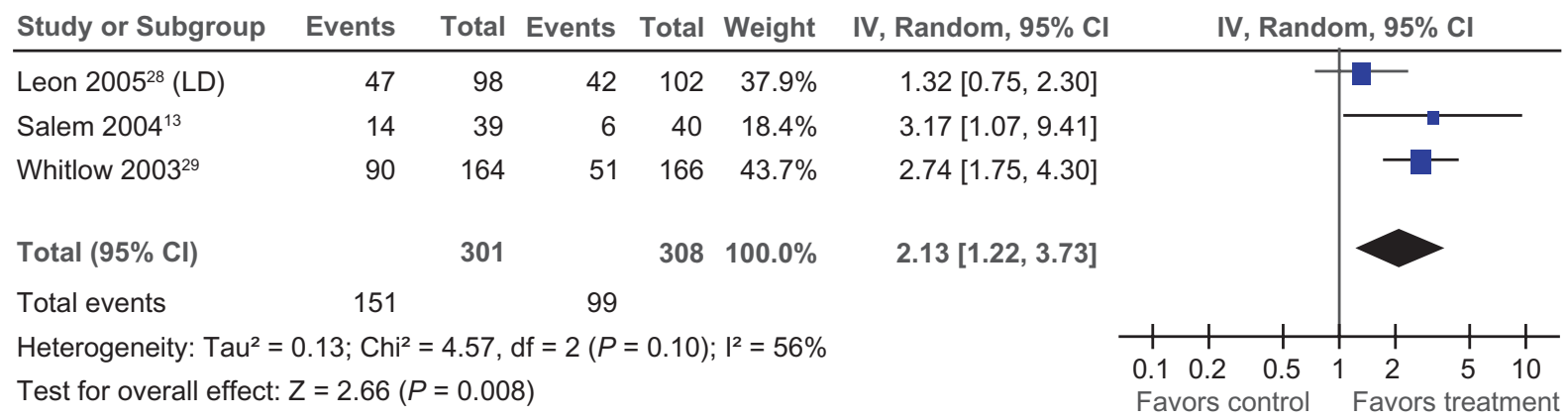

Figure 2 Comparison of PMLR versus maximal medical therapy, outcome CCS Class.

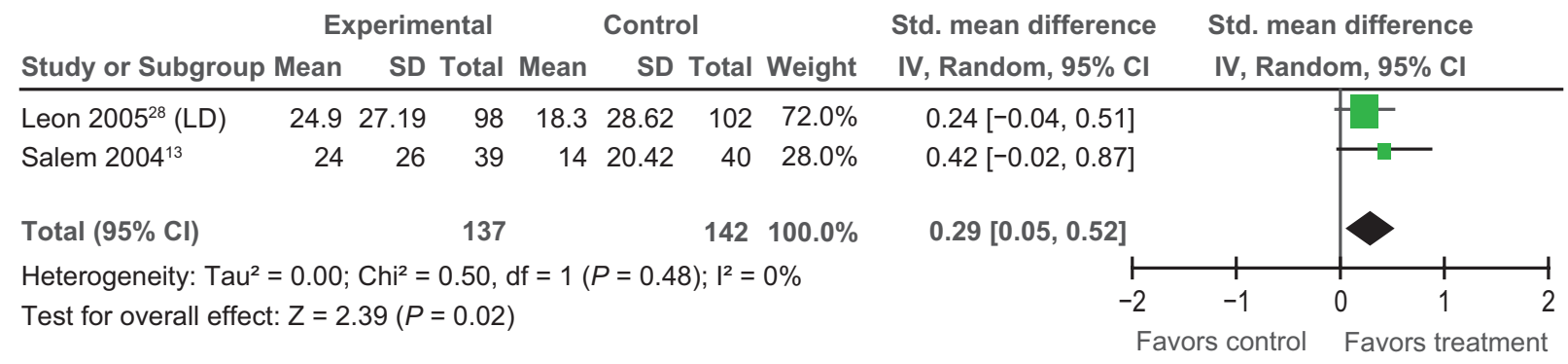

Figure 3 Comparison of PMLR versus maximal medical therapy, outcome angina frequency (AF).

\begin{tabular}{|c|c|c|c|c|c|c|c|c|c|c|}
\hline \multirow{3}{*}{$\frac{\text { Study or Subgroup }}{\text { Leon } 2005^{28} \text { (LD) }}$} & \multicolumn{3}{|c|}{ Experimental } & \multicolumn{3}{|c|}{ Control } & & \multirow{2}{*}{$\begin{array}{l}\text { Std. mean difference } \\
\text { IV, Random, } 95 \% \mathrm{Cl}\end{array}$} & \multirow{2}{*}{\multicolumn{2}{|c|}{$\begin{array}{l}\text { Std. mean difference } \\
\text { IV, Random, } 95 \% \mathrm{CI}\end{array}$}} \\
\hline & Mean & SD & Total & Mean & SD & Total & Weight & & & \\
\hline & 30.9 & 24.09 & 98 & 21.5 & 23.62 & 102 & $71.6 \%$ & $0.39[0.11,0.67]$ & & \\
\hline Salem $2004^{13}$ & 15 & 20.08 & 39 & 8 & 22.27 & 40 & $28.4 \%$ & $0.33[-0.12,0.77]$ & & \\
\hline Total $(95 \% \mathrm{Cl})$ & & & 137 & & & 142 & $100.0 \%$ & $0.37[0.14,0.61]$ & & \\
\hline \multicolumn{8}{|c|}{ Heterogeneity: $\mathrm{Tau}^{2}=0.00 ; \mathrm{Chi}^{2}=0.06, \mathrm{df}=1(P=0.81) ; I^{2}=0 \%$} & & & \\
\hline \multicolumn{8}{|c|}{ Test for overall effect: $Z=3.09(P=0.002)$} & -2 & $\begin{array}{l}-1 \\
\text { rs control }\end{array}$ & $\begin{array}{lc}0 & 1 \\
\text { Favors tre }\end{array}$ \\
\hline
\end{tabular}

Figure 4 Comparison of PMLR versus maximal medical therapy, outcome disease perception (DP).

$$
\text { Experimental Control Std. mean difference Std. mean difference }
$$

Study or Subgroup Mean SD Total Mean SD Total Weight IV, Random, $95 \% \mathrm{Cl} \quad \mathrm{IV}, \mathrm{Random}, 95 \% \mathrm{Cl}$

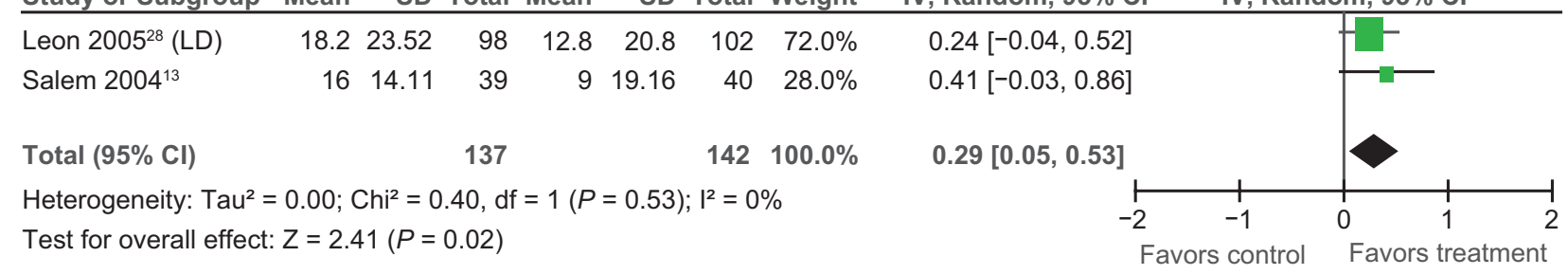

Figure 5 Comparison of PMLR versus maximal medical therapy, outcome physical limitation (PL).

$$
\text { Experimental Control Std. mean difference Std. mean difference }
$$

Study or Subgroup Mean SD Total Mean SD Total Weight IV, Random, $95 \%$ Cl $\quad$ IV, Random, $95 \%$ Cl

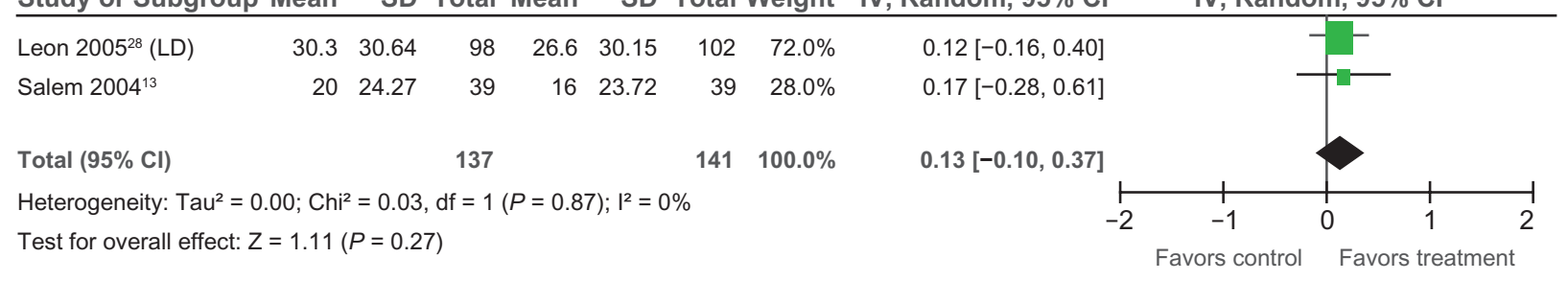

Figure 6 Comparison of PMLR versus maximal medical therapy, outcome angina severity (AS). 


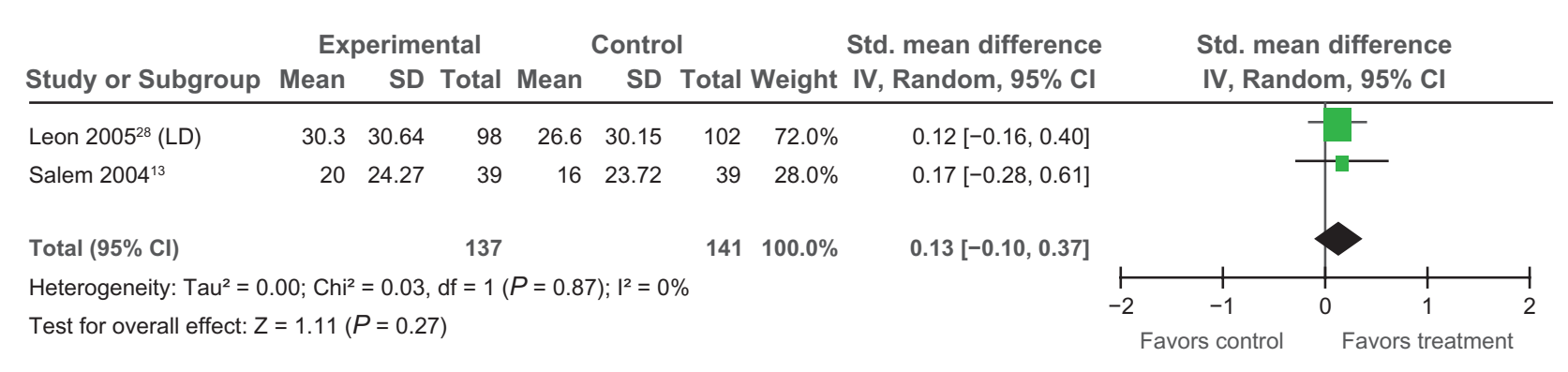

Figure 7 Comparison of PMLR versus maximal medical therapy, outcome treatment satisfaction (TS).

Notably, neither PMLR nor TMLR ${ }^{14}$ has been found to significantly improve SAQ angina frequency or treatment satisfaction scores. A plausible explanation for discordance between observed improvements in CCS class and lack of improvements in SAQ-angina frequency scores may lie with the psychometric properties of the SAQ angina frequency subscale. One of two items comprising this subscale asks patients to recall how many times in the past 4 weeks they have had to take sublingual nitroglycerin for chest pain. This item would be irrelevant to most patients included in these trials who are on maximally-tolerated oral or transcutaneous long-acting nitrate preparations (with nitrate-free intervals to minimize tolerance). A focus on short-acting sublingual nitroglycerine preparations may therefore render the angina frequency subscale insensitive to more global symptomrelated improvements, such as those captured by the CCS scale, for RFA patients following laser therapy.

Lack of significant improvements in SAQ treatment satisfaction scores may also be a measurement issue. The treatment satisfaction subscale comprises 3 items, one of which is oriented toward patient satisfaction with physicianrelated care. ${ }^{42}$ At the individual trial level, care may have been delivered by more than one physician and/or provided by other health care professionals during the course of study follow-up. Such variations would therefore introduce loss of precision in the measurement of potential improvements in patient-physician rapport following intervention.
We found that PMLR did not significantly improve exercise performance as measured by exercise duration in seconds. However, extractable data were combined from three trials, each with different approaches to measurement including the modified Bruce protocol, ${ }^{28}$ the Naughton protocol, ${ }^{29}$ and treadmill or bicycle ergometry. ${ }^{13}$ Inconsistency in measurement across trials may therefore have been a contributing factor, suggesting a need for caution in the interpretation of this finding. Interestingly, Brione et $\mathrm{al}^{14}$ also found that divergent approaches to measuring exercise performance, across primary trials, were problematic in their review of TMLR, with negative results.

With respect to all-cause mortality, our pooling of available data from 4 trials showed that PMLR did not have a significant overall impact. This finding is notable because PMLR yields comparable symptom relief to TMLR, yet TMLR was found by Brione et $\mathrm{al}^{14}$ to be associated with significant risk of early (30-day) postoperative mortality. Moreover, robust methods across trials included in this review such as blinding of participants, operators, and outcome assessors, and the use of sham procedures suggest that the placebo effect is an unlikely explanation for observed reductions in angina following PMLR. In contrast, Brione et al ${ }^{14}$ attributed a high risk of bias to observed improvements in angina following TMLR due to unmasked outcome assessments across primary trials.

The available data seem to suggest that PMLR is as effective as TMLR and that it poses less risk; this is likely because

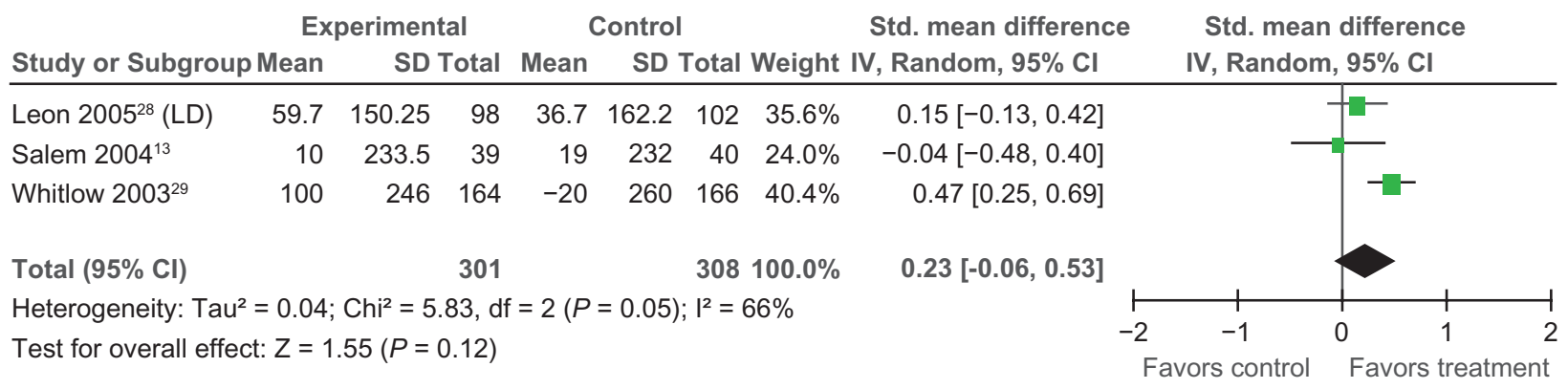

Figure 8 Comparison of PMLR versus maximal medical therapy, outcome exercise duration. 


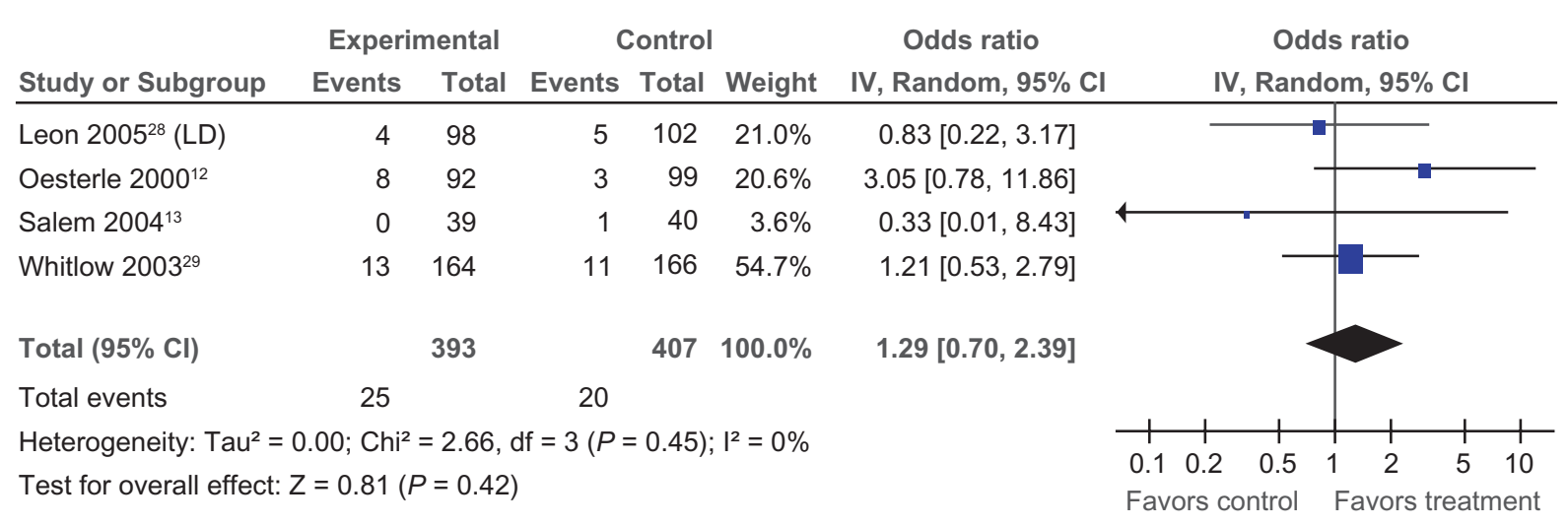

Figure 9 Comparison of PMLR versus maximal medical therapy, outcome all-cause mortality.

PMLR is less invasive. This cannot however be concluded definitively. Lack of detailed reporting on mortality versus adverse events in some trials necessitated our examination of all-cause mortality. The validity of this endpoint as a proxy for the safety of PMLR is uncertain. A more informative and acceptable endpoint would be disease-specific mortality, requiring consistency and greater precision in the assessment of mortality and adverse events in future trials. The incidence and severity of periprocedural risks (eg, pericardial effusion and hematoma, tamponade, and LV and coronary perforation) during PMLR (versus TMLR) should also be examined for a more comprehensive assessment of safety.

The inclusion of a third arm in the DIRECT trial ${ }^{28}$ receiving high-dose laser therapy required us to rerun our primary analyses using data from this high-dose laser group. This was done as a form of sensitivity analysis to determine if data from the high-dose laser group would exert any change in the direction or magnitude of our pooled estimates of effect. Our secondary analyses showed that PMLR had no significant impact across outcomes, including all-cause mortality. Variation in scores between the DIRECT trial $^{28}$ high- and low-dose laser groups were found to be similar, suggesting that these results were due to a true lack of treatment effect.

This unexpected finding raises the question of appropriate laser dose. Across trials, there was a high degree of variability in treatment protocol between patients. The number of transmural channels created (per patient) ranged from $8-35$, and laser pulses per channel ranged from 1-3; the systems used to direct laser energy also differed. The contradictory findings between our primary and secondary meta-analyses suggest an unclear relationship between laser dosage and treatment effect. Further work to clarify the underlying mechanisms of PMLR may shed light on this relationship and the related implications for appropriate laser dosing.

\section{Summary and implications}

Refractory angina patients suffer severely impaired HRQL and have limited options. This review aimed to assess the effects of PMLR versus optimal medical treatment for improving pain symptoms, HRQL, and exercise performance in patients with RFA. While our primary analyses, based on robust trials, showed promising results for improvement in

Table 3 Secondary analyses: comparison of PMLR versus maximal medical therapy for all outcomes using data from the DIRECT trial high-dose group

\begin{tabular}{|c|c|c|c|c|}
\hline Variable & $\begin{array}{l}\text { Pooled standardized } \\
\text { mean difference }\end{array}$ & Pooled odds ratio & $\begin{array}{l}95 \% \mathrm{Cl} \text { for } \\
\text { difference }\end{array}$ & $P$-value \\
\hline CCS class & - & 1.95 & {$[0.90,4.23]$} & 0.09 \\
\hline \multicolumn{5}{|l|}{ Self-reported HRQL } \\
\hline$S A Q-A F$ & 0.18 & _ & {$[-0.20,0.57]$} & 0.35 \\
\hline$S A Q-A S$ & 0.01 & - & {$[-0.23,0.24]$} & 0.95 \\
\hline$S A Q-D P$ & 0.10 & - & {$[-0.25,0.45]$} & 0.57 \\
\hline$S A Q-P L$ & 0.01 & - & {$[-0.23,0.24]$} & 0.95 \\
\hline$S A Q-T S$ & -0.005 & - & {$[-0.30,0.20]$} & 0.72 \\
\hline Exercise duration & 0.17 & _ & {$[-0.18,0.53]$} & 0.97 \\
\hline All-cause mortality & _- & 1.34 & {$[0.44,4.03]$} & 0.61 \\
\hline
\end{tabular}

Abbreviations: AF, angina frequency; AS, angina stability; CCS, Canadian Cardiovascular Society; DP, disease perception; HRQL, health-related quality of life; PL, physical limitation; SAQ, Seattle Angina Questionnaire; TS, treatment satisfaction. 
CCS angina class and aspects of HRQL, key questions about PMLR remain unanswered. Our data suggest that PMLR is a safe treatment option. However, future work should take a more detailed approach to a) patient survival versus the occurrence of adverse events, and b) disease-specific mortality in order to make more definitive conclusions about safety. Clarity is also needed regarding the underlying mechanisms of PMLR in order to gain a better understanding of the relationship between laser dose and the observed benefits of treatment for RFA patients.

\section{Acknowledgments}

This review was funded by the Canadian Institutes of Health Research. We would like to acknowledge Ms Melanie Browne, BHSC, MLIS, Information Specialist, for external audit and replication of our search strategy.

\section{Disclosure}

The authors report no conflicts of interest in this work.

\section{References}

1. Mannheimer C, Camici P, Chester MR, et al. The problem of chronic refractory angina; report from the ESC Joint Study Group on the treatment of refractory angina. Eur Heart J. 2002;23:355-370.

2. Bhatt AB, Stone PH. Current strategies for the prevention of angina in patients with stable coronary artery disease. Curr Opin Card. 2006; 21:492-502.

3. McGillion M, L'Allier P, Arthur H, et al. Recommendations for advancing the care of Canadians living with refractory angina pectoris: A Canadian Cardiovascular Society position statement. Can J Cardiol. 2009; 25:399-401.

4. Henry TD, Satran D, Johnson RK, et al. Natural history of patients with refractory angina. J Am Coll Cardiol. 2006;47:231A.

5. Brorsson B, Bernstein SJ, Brook RH, Werko L. Quality of life of patients with chronic stable angina before and 4 years after coronary artery revascularization compared with a normal population. Heart. 2002; 87:140-145.

6. Erixson G, Jerlock M, Dahlberg K. Experiences of living with angina pectoris. Nurs Sci Res Nordic Countries. 1997;17:34-38.

7. Andrell P, Ekre O, Wahborg P, Eliasson T, Mannheimer C. Quality of life in patients with refractory angina pectoris. International Association for the Study of Pain 11th World Congress on Pain [abstract]; 2005 Aug 21-26; Sydney, Australia: IASP Press; 2005. p. 200.

8. McGillion M, Watt-Watson J, LeFort S, Stevens B. Positive shifts in the perceived meaning of cardiac pain following a psychoeducation for chronic stable angina. Can J Nurs Res. 2007;39:48-65.

9. Chow C-M, Donovan L, Manuel D, et al. Regional variation in selfreported heart disease prevalence in Canada. Can J Cardiol. 2005; 21:1265-1271.

10. Thadani U. Recurrent and refractory angina following revascularization procedures in patients with stable angina pectoris. Coron Artery Dis. 2004; 15 Suppl 1:S1-S4.

11. Oesterle SN. Laser percutaneous myocardial revascularization. Am J Cardiol. 1999;83:46-52.

12. Oesterle SN, Sanborn TA, Ali N, et al. Percutaneous transmyocardial laser revascularization for severe angina: The PACIFIC randomised trial. Lancet. 2000;356:1705-1710.
13. Salem M, Rotevatn S, Stavnes S, et al. Usefulness and safety of percutaneous myocardial laser revascularization for refractory angina pectoris. Am J Cardiol. 2004;93:1086-1091.

14. Briones E, Lacalle JR, Marin I. Transmyocardial laser revascularization versus medical therapy for refractory angina. Cochrane Database of Systematic Reviews 2009, Issue 1. Art. No.: CD003712. DOI:10.1002/14651858.CD003712.pub2.

15. Kohmoto T, Fisher PE, Gu A, et al. Physiology, histology and 2-week morphology of acute transmyocardial laser channels made with a CO2 laser. Ann Thorac Surg. 1997;63:1275-1283.

16. Gassler N, Wintzer HO, Stubbe HM, et al. Transmyocardial laser revascularization. Histological features in human nonresponder myocardium. Circulation. 1997;95:371-375.

17. Burkoff D, Fisher PE, Apefelbaum M, et al. Histologic appearance of transmyocardial laser channels after $4 \frac{1}{2}$ weeks. Ann Thorac Surg. 1996;62:1501-1508.

18. Mueller XM, Tevaearai HH, Genton CY, et al. Transmyocardial laser revascularization in acutely ischaemic myocardium. Eur J Cardiothorac Surg. 1998;13:170-175.

19. Mirhoseini M, Fisher J, Clayton M. Myocardial revascularization by laser: a clinical report. Lasers Surg Med. 1983;3:241-245.

20. Mukherjee D, Bhatt DL, Roe MT, Patel V, Ellis SG. Direct myocardial revascularization and angiogenesis - how many patients might be eligible? Am J Cardiol. 1999:84:589-600.

21. Weimer M, Butz T, Weilepp J, Fassbender, D, Buchert W. Improvement of myocardial perfusion detected by positron emission tomography in patients with end-stage coronary artery disease treated with percutaneous myocardial laser revascularization (abstr).Circulation. 2001;104 suppl II:11-445.

22. Rimoldi O, Burns SM, Rosen SD, et al. Measurement of myocardial blood flow with positron emission tomography before and after transmyocardial laser revascularization. Circulation. 1999;100:2134-2138

23. Burns SM, Brown S, White CA, et al. Quantitative analysis of myocardial perfusion changes with transmyocardial laser revascularization. Am J Cardiol. 2001;87:861-867.

24. Guzetti S, Colombo A, Piccaluga E, et al. Absence of clinical signs of denervation after percutaneous myocardial laser revascularization. Int J Cardiol. 203:91:129-135.

25. Al-Sheikh T, Allen KB, Straka SP, et al. Cardiac sympathetic denervation after transmyocardial laser revascularization. Circulation. 1999; 100:135-140.

26. Myers J, Osterle SN, Jones J, et al. Do transmyocardial and percuatneous laser revascularization induce silent ischemia? An assessment by exercise testing. Am Heart J. 2002;143:1052-1057.

27. Beek J, van der Sloot JAP, Huikeshoven M, et al. Cardiac denervation after clinical transmyocardial laser revasacularization: Short-term and long-term iodine 123-labeled meta-iodobenzylguanide scintigraphic evidence. J Thorac Cardiovasc Surg. 2004;127:517-524.

28. Leon MB, Kornowski R, Downey E, et al. A blinded-randomized, placebo-controlled trial of percutaneous laser myocardial revascularization to improve angina symptoms in patients with severe coronary disease. J Am Coll Cardiol. 2005;46:1812-1819.

29. Whitlow, P1, DeMaio SJ, Perin EC, et al. One-year results of percutaneous myocardial revascularization for refractory angina pectoris. Am J Cardiol. 2003;91:1342-1346.

30. Gray T, Burns SM, Clarke SC, et al. Percutaneous myocardial laser revascularization in patients with refractory angina pectoris. $\mathrm{Am} \mathrm{J}$ Cardiol. 2003;91:661-666.

31. Salem M, Rotevatn S, Nordrehaug JE. Long-term results following percutaneous myocardial laser therapy. Coron Artery Dis. 2006;17: 385-390.

32. McNab D, Khan SN, Sharples LD, et al. An open label, single centre, randomized trial of spinal cord stimulation vs percutaneous myocardial laser revascularization in patients with refractory angina pectoris: the SPiRiT trial. Eur J Cardiol. 2006;27:1048-1053. 
33. Higgins JPT, Green S (editors). Cochrane Handbook for Systematic Reviews of Interventions Version 5.0.2 [updated September 2009]. The Cochrane Collaboration, 2009. Available from www.cochranehandbook.org.

34. Hozo SP, Djulbegovic B, Hozo I. Estimating the mean and variance from the median, range, and the size of a sample. BMC Medical Res Methodol. 2005;5:13 doi:10.1186/1471-2288-5-13.

35. Tomlinson G, Beyene J. [P148] Imputing summary statistics for metaanalysis of continuous data. 12th Cochrane Colloquium, Ottawa, 2-6 Oct 2004.

36. Cohen J. Statistical power analysis for the behavioral sciences. 2nd ed. Hillsdale: Lawrence Earlbaum Associates; 1988.

37. Juni P, Altman DG, Egger M. Systematic reviews in health care: Assessing the quality of controlled clinical trials. BMJ. 2001;323;42-46.

38. DerSimonian R, Laird N. Meta-analysis in clinical trials. Control Clin Trials. 1986;7:177-188.
39. Higgins JP, Thompson SG, Deeks JJ, Altman DG. Measuring inconsistency in meta-analyses. BMJ. 2003;327:557-560.

40. Rucker G, Schwarzer G, Carpenter JR, Schumacher M. Undue reliance on $\mathrm{I}^{2}$ in assessing heterogeneity may mislead. BMC Medical Res Methodol. 2008;8:79.

41. Stone GW, Teirstein PS, Rubenstein R, et al. A prospective, multicentre, randomized trial of percutaneous tranmyocardial laser revascularization in patients with nonrecanalizable chronic total occlusions. $J$ Am Coll Cardiol. 2002;39:1581-1587.

42. Spertus JA, Winder JA, Dewhurst TA, et al. Development and evaluation of the Seattle Angina Questionnaire: A new functional status measure for coronary artery disease. J Am Coll Cardiol. 1995;25:333-341.
Vascular Health and Risk Management

\section{Publish your work in this journal}

Vascular Health and Risk Management is an international, peerreviewed journal of therapeutics and risk management, focusing on concise rapid reporting of clinical studies on the processes involved in the maintenance of vascular health; the monitoring, prevention and treatment of vascular disease and its sequelae; and the involvement of

\section{Dovepress}

metabolic disorders, particularly diabetes. This journal is indexed on PubMed Central and MedLine. The manuscript management system is completely online and includes a very quick and fair peer-review system, which is all easy to use. Visit http://www.dovepress.com/ testimonials.php to read real quotes from published authors.

Submit your manuscript here: http://www.dovepress.com/vascular-health-and-risk-management-journal 